Article

\title{
Anti-allergic effects of fertilized red ginseng marc on 2,4-dini- trochlorobenzene-induced atopic dermatitis-like mice model
}

\author{
Yeun Soo Jung 1,, Jae Young Choi 2,, Young-Sam Kwon ${ }^{1}$, Gyu-Ryeul Park ${ }^{3}$, VinayKumar Dachuri ${ }^{4}$, Young Woo \\ Kim ${ }^{5, *}$, Sae-Kwang Ku ${ }^{3,4, *}$ and Chang-Hyun Song ${ }^{3,4, *}$
}

1 Department of Veterinary Surgery, College of Veterinary Medicine, Kyungpook National University, Daegu, 41566, Republic of Korea; vetsoo1@hanmail.net (Y.S.J.); kwon@knu.ac.kr (Y.S.K.)

2 Department of Urology, College of Medicine, Yeungnam University, Daegu, 42415, Republic of Korea; urocjy@ynu.ac.kr (J.Y.C.)

3 Department of Anatomy and Histology, College of Korean Medicine, Daegu Haany University, Gyeongsan, 38610, Republic of Korea; 9027187@dhu.ac.kr (G.R.P.); gucci200@hanmail.net (S.K.K.); dvmsong@hotmail.com (C.H.S.)

4 Research Center for Herbal Convergence on Liver Disease, Gyeongsan, 38610, Republic of Korea; dachurivinay@gmail.com (VK.D.); gucci200@hanmail.net (S.K.K.); dvmsong@hotmail.com (C.H.S.)

5 School of Korean Medicine, Dongguk University, Gyeongju, 38066, Republic of Korea; ywk@dongguk.ac.kr (Y.W.K.)

* Correspondence: ywk@dongguk.ac.kr (Y.W.K.), gucci200@hanmail.net (S.K.K.) and dvmsong@hotmail.com (C.H.S.); Tel.: +82-31-961-5823 (Y.W.K.); +82-53-819-1549 (S.K.K.); +82-53-819-1822 (C.H.S.); § Y.S.J. and J.Y.C. contributed equally to this.

\begin{abstract}
Atopic dermatitis (AD) is a chronic and allergic skin disease, however, there is no cure for the disease. Many studies have shown anti-AD potentials of red ginseng, while red ginseng marc (RGM) remained after the ginseng extraction is discarded as industrial waste. However, it is recently reported that RGM, particularly fermented RGM (fRGM), still contains the bioactive properties. Thus, anti-allergic effects of fRGM were examined in 2,4-dinitrochlorobenzene-induced AD-like mice model. The model was topically treated with distilled water (control), dexamethasone, or fRGM for six weeks. The treatments of fRGM alleviated the skin lesions and reduced serum IgE levels, compared with the control. The fRGM also reduced skin levels of lipid peroxidation and superoxide anion, however, increased glutathione contents, with down-regulated gene expressions for inflammatory mediators. The histopathological analyses demonstrated that fRGM suppressed the epidermal thickening, collagen deposition, and inflammatory cell and mast cell infiltration, which involved the anti-inflammatory, anti-oxidant, and anti-apoptotic effects. Further, fRGM suppressed hypertrophic changes and inflammatory responses in the spleen and lymph node. The beneficial effects were observed in the dexamethasone and fRGM groups, however, the antioxidant effects were evident only in the fRGM treatments. These provide useful information for developing fRGM as therapeutic sources for AD.
\end{abstract}

Keywords: AD; allergy; skin; DNCB; Panax ginseng; red ginseng marc; fermentation

\section{Introduction}

Atopic dermatitis (AD) is a chronic or chronically relapsing inflammatory skin disease that affects individuals of all ages, from childhood $(15 \%-20 \%)$ to adulthood $(1 \%-$ $3 \%$ ) worldwide [1]. The clinical symptoms are characterized by skin dryness, pruritus, erythema, scaling and eczematous inflammation. The pathogenesis involves imbalanced immune responses to various allergens and defective skin barriers, which elevate immunoglobulin (Ig) E levels and activate T cells, particularly type 2-helper T cells (Th2), dendritic cells, mast cells and eosinophils [2]. Consequently, the allergic reactions stimulate production of Th2-derived cytokines and chemokines, leading to an increase in the release of Th1-derived pro-inflammatory cytokines including tumor necrosis factor (TNF)- $\alpha$, interferon (IFN)- $\gamma$ and various interleukins (IL) families, with elevated immune cell 
infiltrations in the skin lesions [3,4]. The incidence of AD is continuously increasing, posing a public health problem with declining quality of life in the patients and increasing healthcare burden [5]. The treatment options include topical corticosteroids (i.e., dexamethasone), calcineurin inhibitors, antihistamines, antibiotics and phototherapy [6]. However, they are limited to the symptom management, and some of them can be often ineffective or have adverse effects in the long-term use, such as immune suppression, hyperglycemia and skin atrophy [7]. Thus, there is an unmet need to develop safe and effective treatments for AD.

Red ginseng (the steamed root of Panax ginseng C.A. Meyer) is a traditional medicine primarily used to treat various diseases including inflammatory skin diseases in Korea, China and Japan [8]. There have been many reports regarding the anti-allergic effects of red ginseng [9]. Furthermore, topical treatment with red ginseng has shown anti-AD effects in animal models by alleviating skin lesions and reducing IgE levels and production of the relevant inflammatory mediators [10-14]. Clinical studies also support the anti-AD effects by showing improved clinical symptoms following the reduction in serum IgE levels $[15,16]$. In particular, ginsenosides including Rb1, Rb2, Rg3 and Rh1 amongst compounds belonging to red ginseng, are believed to be responsible for the anti-AD effects via immune-modulating and anti-inflammatory pathways $[2,17,18]$.

Red ginseng marc (RGM) is a byproduct of red ginseng extraction, and it is usually treated as an industrial waste. However, increasing demand for the red ginseng among patients finding more effective medications has aroused interests in the recycling of RGM. In this context, recent studies have shown evidences supporting the immunomodulatory, anti-inflammatory, antioxidant and anticancer effects of RGM, despite the small contents of bioactive components such as ginsenosides, polyphenols and flavonoids [19-22]. Furthermore, the fermentation process of red ginseng and RGM has been reported to enhance the bioactive properties in AD [9]. Fermented RGM contains a greater proportion of ginsenosides, Rg2, Rg3 and Rh2 than the non-fermented RGM, which enhances its antioxidant activities and incidental favorable effects $[23,24]$. Our previous study has shown that topical treatment of RGM extracts fermented with a complex of microorganisms promotes hair growth [25]. The fermented RGM contained crude ginsenosides (1.7 mg/g) and polyphenols $(9.8 \mathrm{mg} / \mathrm{g})$, and the antioxidant activities were more than those of non-fermented form. The detected ginsenosides were as following: Rb1 (0.6 mg/kg), Rb2 (0.9 mg/kg), Rb3 $(1.8 \mathrm{mg} / \mathrm{kg}), \operatorname{Rc}(1.9 \mathrm{mg} / \mathrm{kg}), \operatorname{Rd}(3.5 \mathrm{mg} / \mathrm{kg}), \operatorname{Re}(3.1 \mathrm{mg} / \mathrm{kg}), \operatorname{Rf}(3.9 \mathrm{mg} / \mathrm{kg}), \operatorname{Rg} 2$ (12.1 $\mathrm{mg} / \mathrm{kg}), \operatorname{Rg} 3(9.6 \mathrm{mg} / \mathrm{kg}), \operatorname{Rh} 1(17.9 \mathrm{mg} / \mathrm{kg})$. Thus, anti-allergic effects of the fermented RGM were examined in 2,4-dinitrochlorobenzene (DNCB)-induced AD-like mice model.

\section{Materials and Methods}

\subsection{Reagents and antibodies}

Dexamethasone, DNCB, o-phthalaldehyde, and nitroblue tetrazolium (NBT) were purchased from Sigma-Aldrich (St. Louis, MO, USA). The following antibodies were used: rabbit antibodies against cleaved caspase-3 (Cas-3, \#9661) and cleaved poly(ADP-ribose) polymerase (PARP; \#9545) from Cell Signaling Technology (Danvers, MA, USA); rabbit antibodies against 4-hydroxynonenal (4-HNE; \#ab46545) and matrix metalloprotease (MMP)-9 (\#ab38898) from Abcam (Cambridge, UK); rabbit antibody against nitrotyrosine (NT) from Millipore Corp. (\#06-284, Billerica, CA, USA); rabbit antibodies against inducible nitric oxide synthase-2 (iNOS; \#sc-651) and IL-1 $\beta$ (\#sc-7884), and mouse antibody against TNF- $\alpha$ (\#sc-52746) from Santa Cruz Biotechnology (Santa Cruz, CA, USA); goat antibodies against IFN- $\gamma$ (\#AF781) and IL-2 (\#BAF1815) from R\&D System (Minneapolis, MN, USA).

\subsection{Preparation of fermented RGM}

Fermented RGM was provided by Punggi Ginseng Farming Corp. (Yeongju, Korea), and used as same lot number as that used in our previous study [25]. Briefly, 6-year-old Korean red ginseng was extracted by boiling in distilled water (DW) for $3 \mathrm{~h}$ at $80^{\circ} \mathrm{C}$, and 
the remaining RGM was dried and grinded. The RGM was fermented with the effective microorganisms containing Lactobacillus casei, Saccharomyces cerevisiae and Rhodobacter capsulatus (Ever Miracle ${ }^{\mathrm{TM}}$, Ever Miracle Co., Ltd., Jeonju, Korea) at $45^{\circ} \mathrm{C}$ for 15 days, and extracted by boiling three times in DW for $3 \mathrm{~h}$ at $80^{\circ} \mathrm{C}$. Then, the extracts (fRGM) were lyophilized, and the yield efficiency was determined to be $40.5 \%$. The ginsenoside components (Rg1, Re, Rf, Rg2, Rh1, Rb1, Rc, Rb2, Rb3, Rd, Rg3, and Rh2), sugars and polysaccharides were analyzed using an Agilent 1100 HPLC system with an Agilent C18 column under the corresponding standard curves. The fRGM was dissolved in DW, and stored at $4{ }^{\circ} \mathrm{C}$ until use.

\subsection{Animals}

All animal experiments were conducted according to the national regulations of the usage and welfare of laboratory animals, and approved by the Institutional Animal Care and Use Committee of Daegu Haany University (Approval No.: DHU2015-020). Six-weekold female SKH-1 hairless mice were obtained from Orient Bio Inc. (Seongnam, Korea). Animals were housed in a temperature $\left(20-25^{\circ} \mathrm{C}\right)$ - and humidity $(50 \%-55 \%)$-controlled room with a light / dark cycle of $12 / 12 \mathrm{~h}$. Feed and water were provided ad libitum.

\subsection{Induction of AD-like lesions and treatments}

After two-week acclimatization, mice were divided into six groups ( $\mathrm{n}=8$ / group): one normal (Intact) and five AD-like model groups. The AD-like model was induced by DNCB dissolved in a mixture of acetone and olive oil (3:1) as a vehicle, as described previously [26]. Briefly, 1\% DNCB was topically applied to the dorsal skin daily for a week, followed by challenging with $0.5 \%$ DNCB three times a week for four weeks. The model was topically treated with DW (AD Con), dexamethasone at $10 \mathrm{mg} / \mathrm{ml}$ (DEXA), or fRGM at 400, 200 and $100 \mathrm{mg} / \mathrm{ml}$ (fRGM400, fRGM200 and fRGM100, respectively), daily for further six weeks. The Intact group was applied by the vehicle without DNCB, and treated with DW. All topical treatments were applied in a volume of $200 \mu$. The mice were euthanized using $\mathrm{CO}_{2}$ gas after all treatments, and the bloods and tissues of the skin lesion, spleens and left submandibular lymph nodes were collected.

\subsection{Gross assessment of skin severity and scratching}

Skin severity and scratching were assessed by a trained observer blinded to the groups, as described previously [26,27]. Skin severity was scored as 0 (no symptoms), 1 (mild), 2 (moderate), and 3 (severe) for pruritus/itching, erythema/hemorrhage, edema, excoriation/erosion and scaling/dryness. Scratching was monitored for $30 \mathrm{~min}$ as follows: one scratching motion was defined as one or more back-and-forth motions of the hind paw directed toward contacting the dorsal area, ending with either licking or placing back the hind paw on the floor.

\subsection{Assessment of total serum IgE and splenic cytokines}

Serum IgE and splenic cytokines were assessed using enzyme-linked immunosorbent assay (ELISA) kits (BD Bioscience, San Jose, CA, USA, for IgE and TNF- $\alpha$; MyBioSource, Inc., San Diego, CA, USA, for IL-1 $\beta$ and IL-10), as described previously [26]. Splenic cytokines were normalized to the protein content. All levels were examined in duplicate using standard curves with a plate reader (Tecan, Männedorf, Switzerland).

\subsection{Antioxidant activities}

Skin levels of glutathione (GSH), malondialdehyde (MDA) and NBT reduction were determined, as described previously [26]. For GSH, skin homogenates were mixed with $30 \%$ trichloroacetic acid, and centrifuged. The supernatant was incubated with $1 \mathrm{mg} / \mathrm{mL}$ o-phthalaldehyde for $15 \mathrm{~min}$, and measured using standard curves with a fluorescence spectrophotometer (RF-5301PC, Shimadzu Corp., Tokyo, Japan) (kexc $=350 \mathrm{~nm}$; kem $=420$ $\mathrm{nm})$. For MDA, the homogenates were mixed with $10 \%$ trichloroacetic acid, and centrifuged. The supernatant was incubated with $0.67 \%$ thiobarbituric acid for $15 \mathrm{~min}$ at $100^{\circ} \mathrm{C}$, 
and assessed at 535 and $572 \mathrm{~nm}$. For NBT reduction, the homogenates were incubated with $1 \mathrm{mg} / \mathrm{mL}$ NBT for $1 \mathrm{~h}$ at $37^{\circ} \mathrm{C}$, and the precipitates were solubilized in a mixture of $2 \mathrm{M}$ potassium hydroxide and dimethyl sulfoxide. The reduction of NBT to formazan was measured at $600 \mathrm{~nm}$.

\subsection{Reverse transcription-quantitative polymerase chain reaction ( $R T-q P C R$ )}

Total RNA from skin tissue was extracted using TRIzol reagent (Invitrogen, Carlsbad, CA, USA), as described previously [26]. The RNA concentration and quality were determined using a CFX96 ${ }^{\mathrm{TM}}$ Real-Time System (Bio-Rad, Hercules, CA, USA), and the contaminating DNA was removed using recombinant DNase I (Ambion, Austin, TX, USA). The cDNA was processed with a high-capacity cDNA reverse transcription kit (Applied Biosystems, Foster City, CA, USA), and amplified using primers for TNF- $\alpha$, IL-4, IL5 and IL-13 (listed in Table S1). The expression was normalized to that of glyceraldehyde 3-phosphate dehydrogenase (GAPDH).

\subsection{Histopathology and immunohistochemistry}

Histopathology and immunohistochemistry were performed, as described previously [26]. Tissue sample was fixed in 10\% formalin, and paraffin-embedded. The serial sections (at $4 \mu \mathrm{m}$ ) were stained with hematoxylin and eosin (H\&E), Masson's trichrome and toluidine blue. For immunohistochemical analysis, following antigen retrieval, the sections were treated with $3 \% \mathrm{H}_{2} \mathrm{O}_{2}$ in methanol for $30 \mathrm{~min}$, and then with a blocking solution for $1 \mathrm{~h}$. The sections were incubated with primary antibodies against Cas-3 (1:400), TNF- $\alpha$ and NT (1:200), and PARP, 4-HNE, MMP-9, iNOS, IL-1 $\beta$, IL-2 and IFN- $\gamma$ (1:100), overnight at $4{ }^{\circ} \mathrm{C}$. Next day, they were incubated with a biotinylated secondary antibody and ABC reagents (Vector Lab., Burlingame, CA, USA) for $1 \mathrm{~h}$, and visualized using a peroxidase substrate for $3 \mathrm{~min}$. Sections omitting primary antibodies were used as the negative control. The cell or fibers showing more than $30 \%$ of immunoreactivities were regarded as positive. The results were analyzed using an iSolution image analysis program (IMT $i$-solution Inc., Vancouver, BC, Canada) by a histopathologist blinded to the groups.

\subsection{Statistical analysis}

All data are expressed as the mean \pm standard deviation (SD). Homogeneity of variances was examined by the Levene test. Kinetic changes in skin severity, scratching and body weight were examined by two-way analysis of variance (ANOVA) with main factors for the groups and the weeks measured, and the weeks were treated as repeated measurements. The other data were examined by one-way ANOVA. The multi-comparison among the groups was examined by Tukey and Dunnett T3 post-hoc tests in cases of equal and non-equal variances, respectively. The results were described focused on multi-comparisons between the AD Con and fRGM groups. Statistical significance was set at $p<0.05$.

\section{Results}

\subsection{Effects on AD-like skin lesions and serum levels of IgE}

Severe skin lesions with dryness, slime and scab were observed in the AD Con group, however, they were attenuated in the DEXA and fRGM groups, particularly in the higher dose group (Figure 1a). Kinetic changes of the skin severity and scratching behaviors showed significant main effects for the groups and the weeks measured $(p<0.01$, Figure $1 \mathrm{~b}$ and 1c). There were also significant interactions between the groups and weeks $(p<$ $0.01)$. The post-hoc tests revealed significant increases in the severity scores and scratching numbers in the AD Con group compared with the Intact $(p<0.01)$. However, the skin severities were significantly reduced in the DEXA at 1 to 6 weeks post-treatment (WPT), in the fRGM400 and fRGM200 at 2 to 6 WPT, and in the fRGM100 at 3, 4 and 6 WPT, compared with the AD Con $(p<0.05)$. The scratching was reduced in the DEXA at 2 to 6 WPT, in the fRGM400 at 3, 5 and 6 WPT, and in the fRGM200 at 6 WPT $(p<0.05)$. Body 
weights were not different among the groups (Figure 1d). Following all treatments, serum levels of IgE were significantly different among the groups ( $p<0.01$, Figure 1e). The levels were higher in the AD Con group compared than in the Intact; however, they were reduced by $40.8 \%, 65.8 \%, 72.9 \%$ and $81.1 \%$ in the DEXA, fRGM400, fRGM200 and fRGM 100 groups, respectively, compared with the $\mathrm{AD}$ Con $(p<0.05)$.
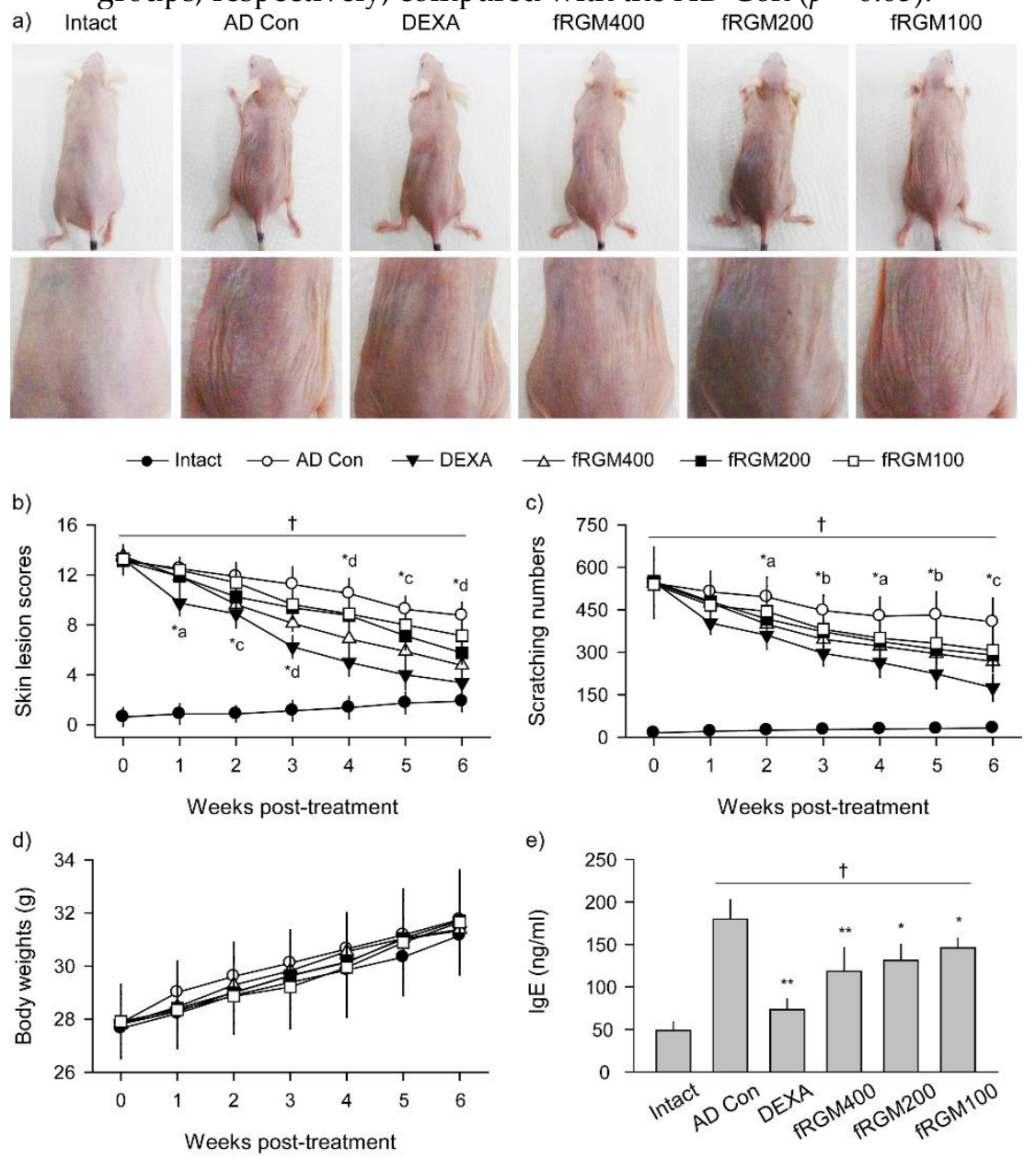

Figure 1. Effects on AD-like symptoms and production of IgE. (a) Representative images for the skin lesions. (b) Skin severity scores. (c) Scratching numbers. (d) Body weight changes. (e) Serum levels of IgE. Values are represented as the means \pm standard deviations (SDs). t: $p<0.05$ vs. the Intact group; ${ }^{* *}: p<0.01$ and ${ }^{*}: p<0.05$ vs. the AD Con. ${ }^{*} \mathrm{a},{ }^{*} \mathrm{~b},{ }^{*} \mathrm{c}$ and ${ }^{*} \mathrm{~d}: p<0.05$ in the DEXA, in the DEXA and fRGM400, in the DEXA, fRGM400 and fRGM200, and in the DEXA and all fRGM groups vs. the AD Con.

\subsection{Effects on skin antioxidant activities and production of inflammatory mediators}

Skin levels of GSH, MDA and NBT reduction were significantly different among the groups ( $p<0.01$, Figure 2a-c). The GSH contents were lower in the AD Con than in the Intact; however, it was increased in the fRGM400 and fRGM200 compared with the AD Con group $(p<0.01)$. Conversely, while the levels of MDA and NBT reduction were increased in the AD Con compared with the Intact, they were reduced in all of the fRGM groups compared with the AD Con $(p<0.05)$. However, the levels for GSH, MDA and NBT were not different between the AD Con and DEXA groups. Skin expressions of mRNA for TNF- $\alpha$, IL-4, IL-5 and IL-13 were also significantly different among the groups $(p<0.01$, Figure $2 \mathrm{~d})$. The expressions were significantly higher in the AD Con than in the Intact $(p<0.05)$; however, the expressions of TNF- $\alpha$, IL-4 and IL- 5 were significantly reduced in the DEXA, fRGM400 and fRGM200, and the IL-13 was reduced in the DEXA and all of the fRGM groups, compared with the AD Con $(p<0.05)$. 

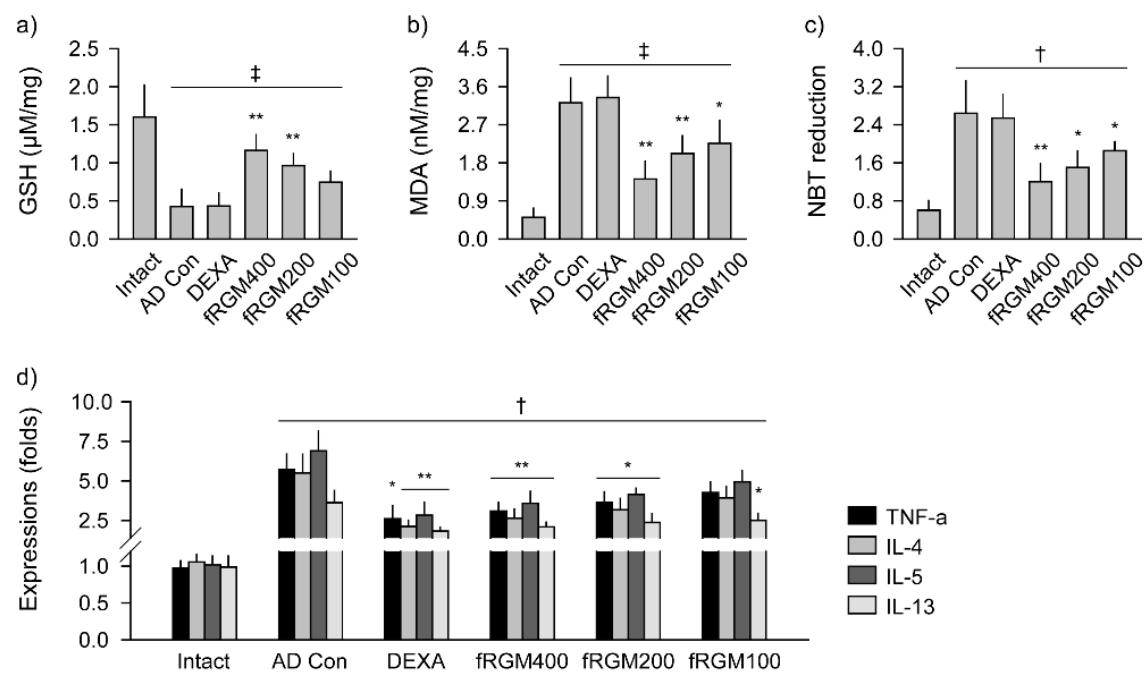

Figure 2. Effects on antioxidant activities and production of inflammatory mediators in skin lesions. (a-c) Levels of glutathione (GSH), malondialdehyde (MDA) and nitroblue tetrazolium (NBT) reduction. (d) Relative mRNA expressions for tumor necrosis factor (TNF)- $\alpha$, interleukin (IL)-4, IL-5 and IL-13 to GAPDH in the skin lesion. Values are represented as the means \pm SDs. $\ddagger$ : $p<0.01$ and $+: p<$ 0.05 vs. the Intact group; ${ }^{* *}: p<0.01$ and ${ }^{*}: p<0.05$ vs. the AD Con.

a) Intact ADCon DEXA fRGM400 fRGM200 fRGM100
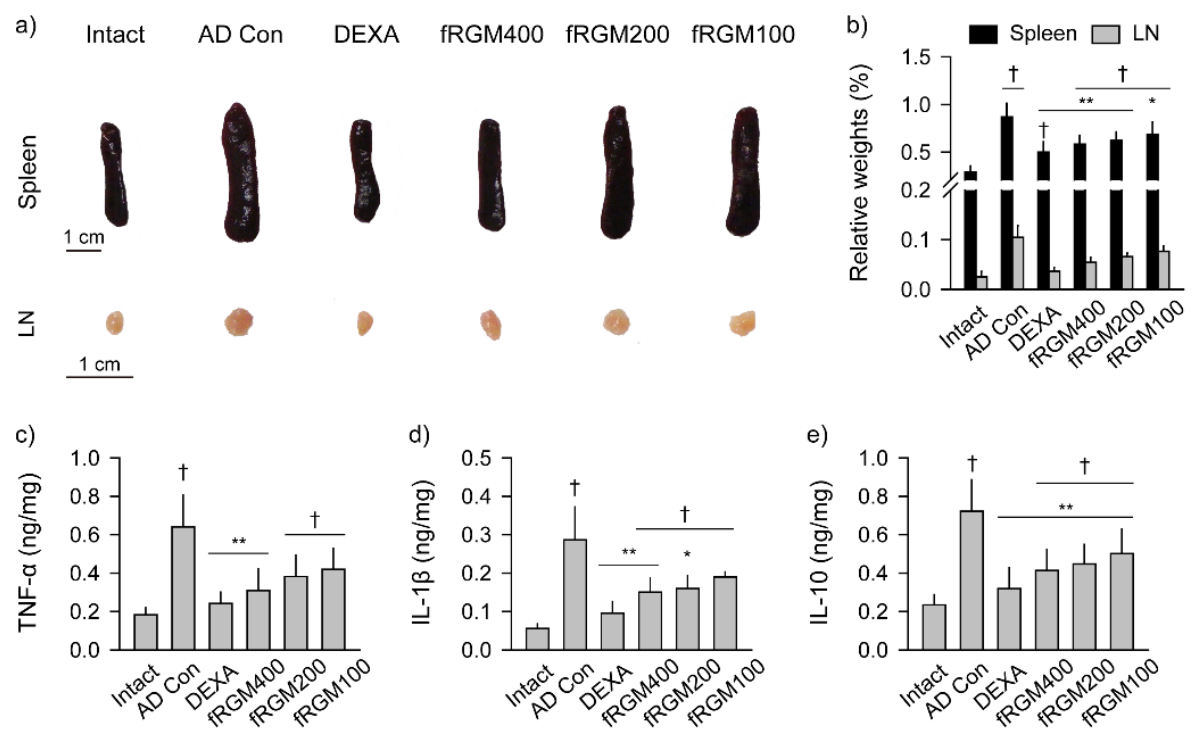

Figure 3. Effects on the lymphoid organs and the production of inflammatory cytokines. (A) Representative images of the spleen and lymph node (LN). (B) Relative weights of the spleen and LN to the body weights. (C-E) Splenic levels of tumor necrosis factor (TNF)- $\alpha$, interleukin (IL)-1 $\beta$ and IL10. Values are represented as the means \pm SDs. $+: p<0.05$ vs. the Intact group; ${ }^{* *}: p<0.01$ and ${ }^{*}: p<$ 0.05 vs. the AD Con.

\subsection{Effects on lymphoid tissues in AD-like model}

The spleen and lymph nodes were enlarged in the AD Con group, while their sizes were relatively reduced in the DEXA and fRGM groups (Figure 3a). The absolute weights of the spleen and lymph node and the relative weights to the body weights were significantly different among the groups ( $p<0.01$, Figures $3 \mathrm{~b}$ and S1). The absolute and relative weights were significantly increased in both organs in the AD Con compared with the Intact $(p<0.01)$. However, the weights were reduced in the spleen of the DEXA and fRGM groups and in the lymph nodes of the DEXA, fRGM400 and fRGM200, compared with the AD Con $(p<0.05)$. The splenic levels of TNF- $\alpha$, IL-1 $\beta$ and IL-10 were significantly different among the groups $(p<0.01$, Figure $3 c-e)$. The levels were significantly higher in the AD Con than in the Intact $(p<0.05)$. However, compared with the AD Con, the TNF- $\alpha$ were reduced in the DEXA and fRGM400; the IL-1 $\beta$ were reduced in the DEXA, fRGM400 and 
fRGM200; the IL-10 were reduced in the DEXA and fRGM groups $(p<0.05)$. There were no significant differences in the TNF- $\alpha$ levels in the DEXA and fRGM400, and in the IL$1 \beta$ and IL-10 levels in the DEXA, compared with those in the Intact group.

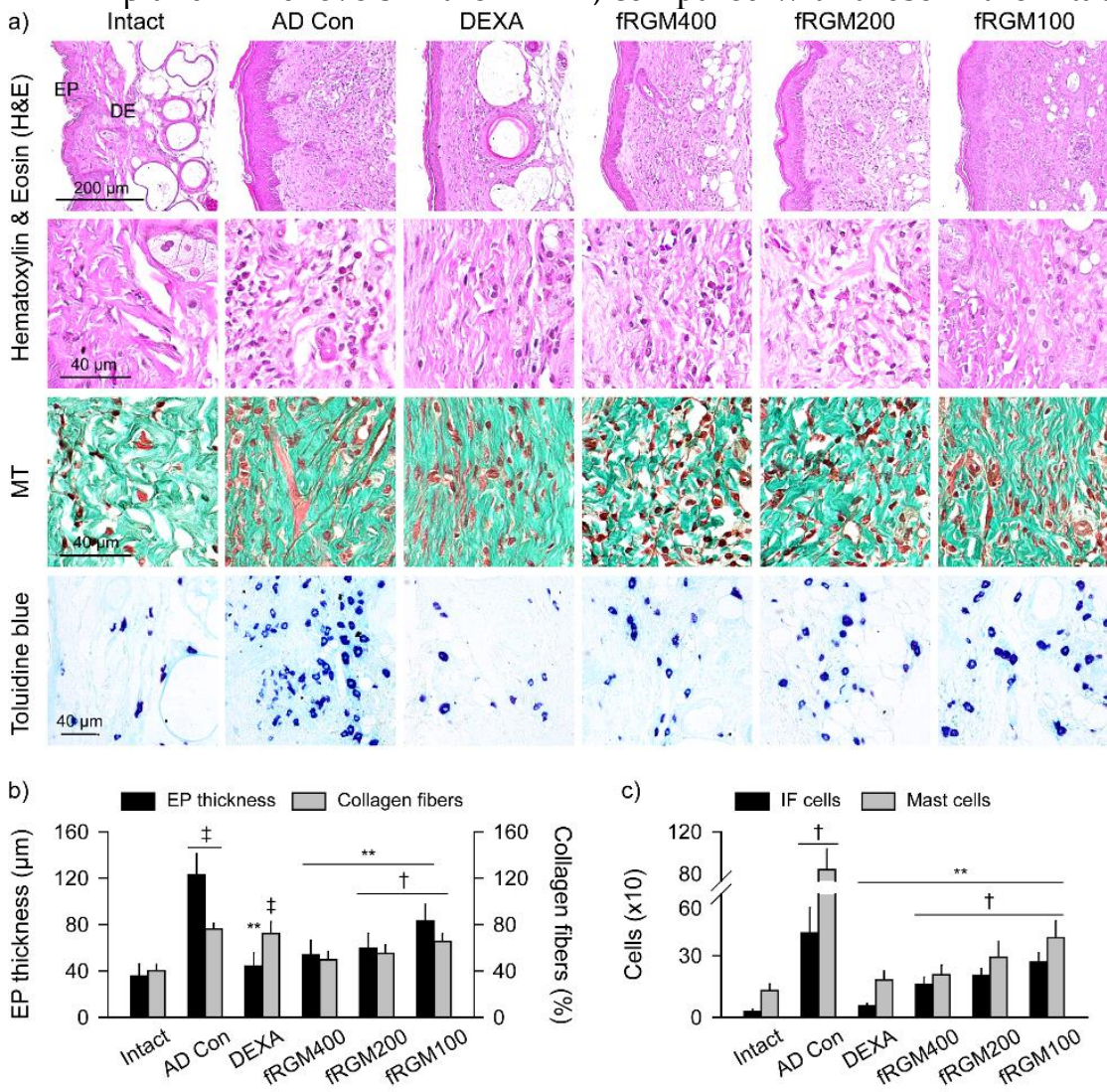

Figure 4. Histopathological changes in AD-like skin lesions. (A) Representative images in stains of H\&E, Masson's trichrome (MT) for collagen deposition and toluidine blue for mast cells. In H\&E stains, the upper shows the epidermis (EP) and dermis (DE), and the lower is the high-magnified DE. (B-C) The EP thickness $(\mu \mathrm{m})$, collagen deposition $\left(\% / \mathrm{mm}^{2}\right)$, number of inflammatory (IF) cells and mast cells in the DE $\left(\times 10\right.$ cells $\left./ \mathrm{mm}^{2}\right)$. Values are represented as the means \pm SDs. $\ddagger: p<0.01$ and $t: p<0.05$ vs. the Intact group; ${ }^{* *}$ : $p<0.01$ vs. the AD Con.

\subsection{Histopathological changes in AD-like skin lesions}

The skin lesions were severe with epidermal thickening, increased dermal infiltration of the inflammatory cells and mast cells, and collagen deposition in the AD Con group; however, they were mild in the DEXA and fRGM groups (Figure 4a). Indeed, the epidermal thickness, collagen areas and the number of inflammatory cells and mast cells were significantly different among the groups ( $p<0.01$, Figure $4 \mathrm{~b}$ and $4 \mathrm{c})$. They were increased in the AD Con compared with the Intact $(p<0.05)$. However, compared with the AD Con, the epidermal thickness and number of inflammatory cells and mast cells were reduced in the DEXA and fRGM groups $(p<0.05)$, and the collagen area was reduced only in the fRGM400 and fRGM200 $(p<0.01)$. There were no differences in the epidermal thickness of the DEXA and fRGM400 and the collagen areas of the fRGM400, compared with those of the Intact. Immunoreactivities for Cas-3, PARP, NT, 4-HNE, MMP-9, IFN- $\gamma$, iNOS, TNF- $\alpha$, IL-1 $\beta$ and IL-2 were evident in the AD Con group; however, they were attenuated in the DEXA and fRGM groups (Figure 5a). The immunostained cells were significantly different among the groups $(p<0.01$, Figure $5 b$ and $5 c)$. The immunostained cells were more in the AD Con than in the Intact; however, they were significantly fewer in the DEXA and fRGM groups than in the AD Con $(p<0.05)$. There were no differences in the PARP-positive cells in the fRGM400, NT- and IL-2-positive cells in the DEXA and fRGM400, IFN- $\gamma$-positive cells in the DEXA, compared with those in the Intact group. 


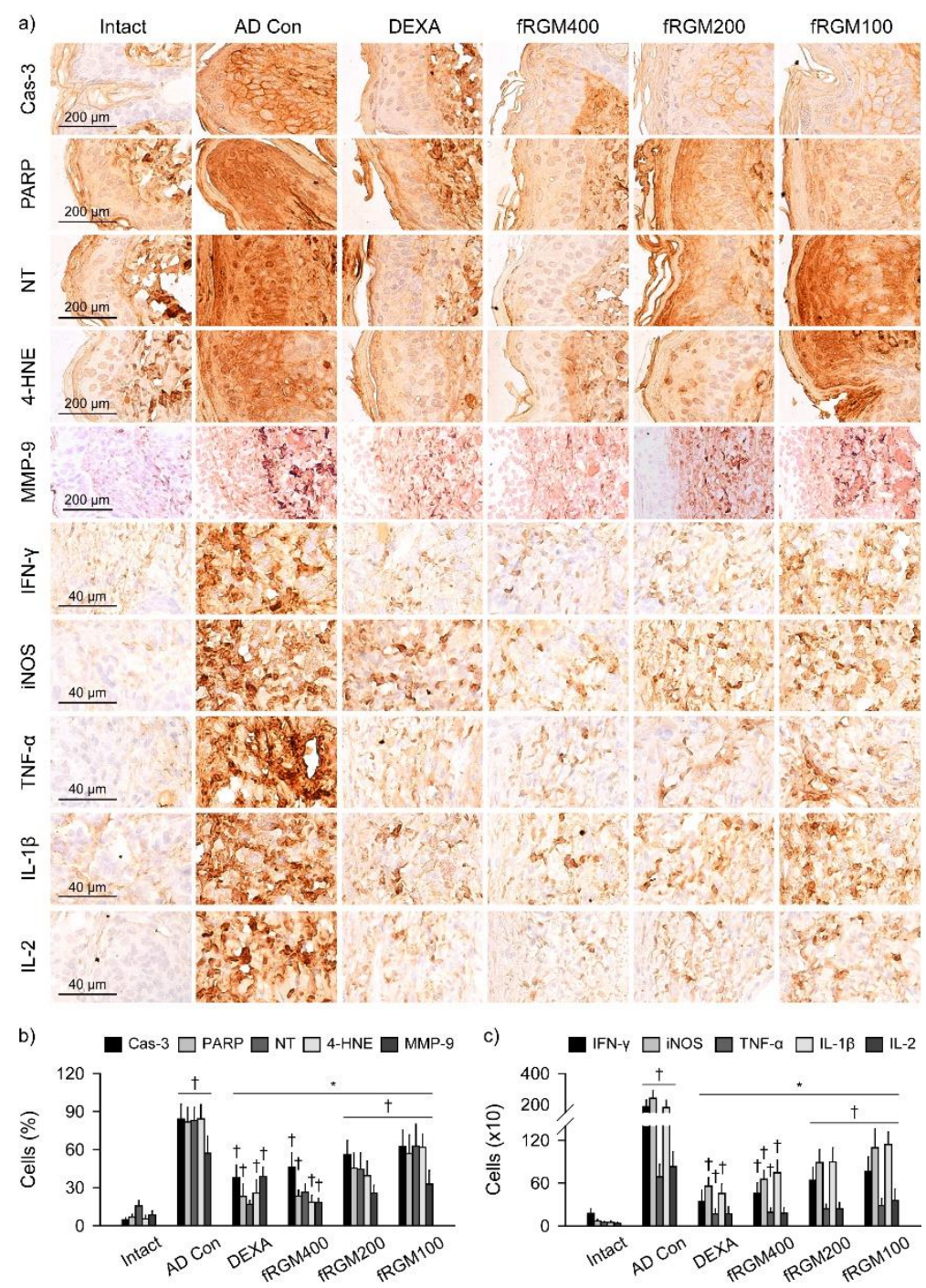

Figure 5. Immunohistochemistry in AD-like skin lesions. (A) Representative images in immunostains for cleaved caspase-3 (Cas-3), cleaved poly(ADP-ribose) polymerase (PARP), nitrotyrosine (NT), 4-hydroxynonenal (4-HNE), matrix metalloprotease (MMP)-9, interferon (IFN)- $\gamma$, inducible nitric oxide synthase-2 (iNOS), TNF- $\alpha$, IL-1 $\beta$ and IL-2. (B-C) The immunoreactive cells in the dermis ( $\%$ in 100 cells or $\times 10$ cells $/ \mathrm{mm}^{2}$ ). Values are represented as the means \pm SDs. $\dagger: p<0.05$ vs. the Intact group; ${ }^{*}: p<0.05$ vs. the AD Con.

\subsection{Histopathological changes in the lymphoid organs of AD-like model}

The AD Con group showed hypertrophic changes in both spleen and lymph nodes (Figures 6a and 7a); however, these changes were attenuated in the fRGM groups (Figure 6a). In the spleen, the thickness in the central region and the number of white pulp cells were significantly different among the groups $(p<0.01$, Figure $6 \mathrm{~b})$; they were increased in the AD Con compared with the Intact, however, reduced in the DEXA and fRGM groups compared with the AD Con $(p<0.05)$. The thickness was not different in the DEXA and fRGM400 compared with the Intact. There were no differences in the red pulp cells among the groups. In the lymph nodes, the total and cortical thickness and the number of follicles in the cortex were also significantly different among the groups $(p<0.05$, Figure $7 b)$; they were increased in the AD Con compared with the Intact, however, reduced in the DEXA and fRGM groups except for the cortical thickness of the fRGM100, compared with the AD Con $(p<0.01)$. In both tissues, the immunoreactivities for IFN- $\gamma$, iNOS, TNF- $\alpha$, IL-1 $\beta$ and IL-2 were evident in the AD Con, however, they were attenuated in the DEXA and fRGM groups. The number of immunostained cells was different among the groups in the spleen and lymph node ( $p<0.01$, Figures $6 \mathrm{c}$ and $7 \mathrm{c})$; it was increased in the AD Con compared with the Intact, however, reduced in the DEXA and fRGM groups except for the 
splenic TNF- $\alpha$ cells of the fRGM100, compared with the AD Con $(p<0.05)$. There were no differences in the immunostaining for IL-1 $\beta$ in the DEXA and fRGM400 and for IL-2 in the DEXA in the spleen, and for IFN- $\gamma$ in the DEXA and for iNOS in the fRGM400 in the lymph node, compared with the observations in the Intact.

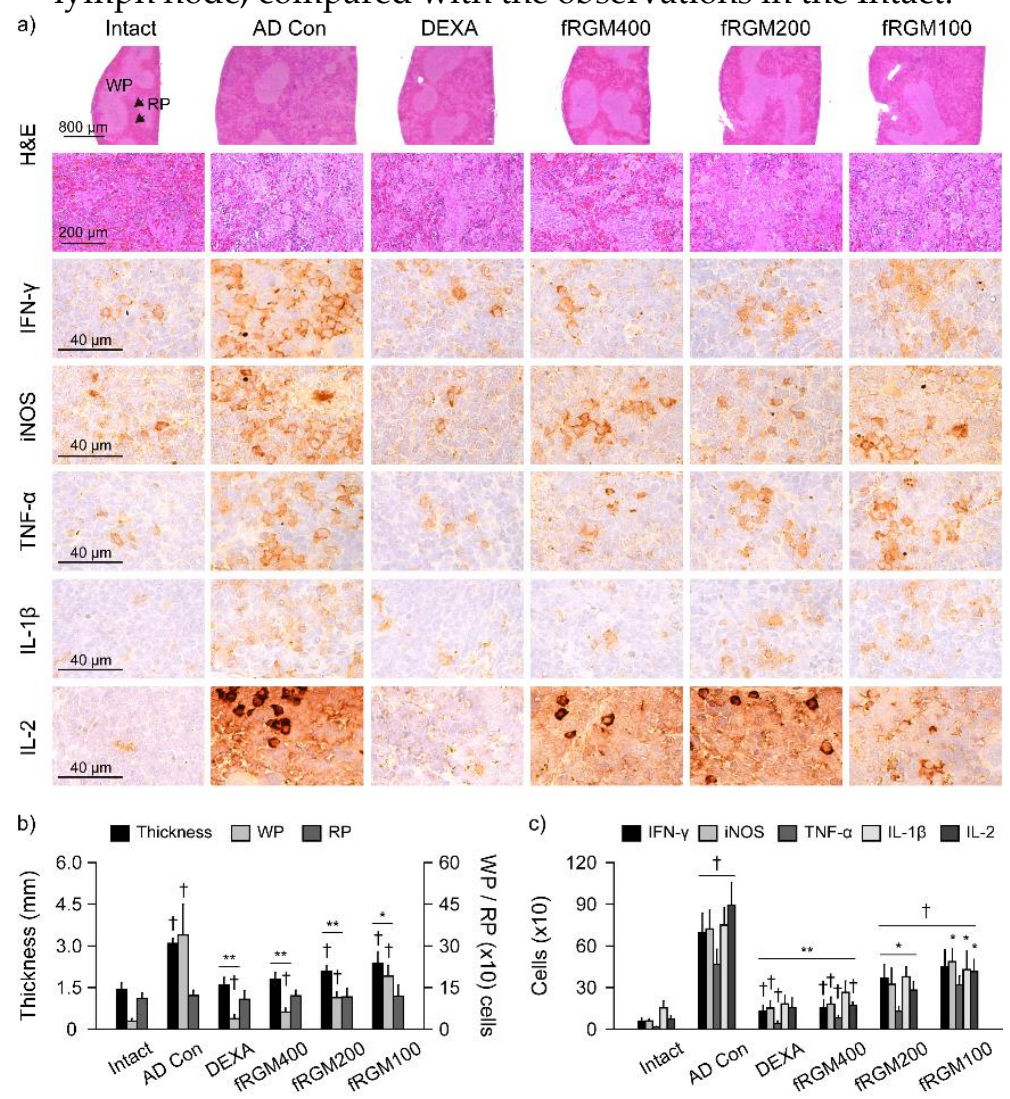

Figure 6. Histopathological changes in the spleen of AD-like model. (A) Representative image in H\&E stain and immunostains for IFN- $\gamma$, iNOS-2, TNF- $\alpha$, IL-1 $\beta$ and IL-2 in the spleen. In H\&E stains, the upper showed white pulp (WP) and red pulp (RP, arrows), and the lower was the high-magnified parenchyma. (B) The splenic thickness in the central region ( $\mathrm{mm}$ ) and number of the WP and RP cells in parenchyma (cells $\left./ \mathrm{mm}^{2}\right)$. (C) Immunoreactive cells in the splenic parenchyma $\left(\times 10\right.$ cells $\left./ \mathrm{mm}^{2}\right)$. Values are represented as the means \pm SDs. $t: p<0.05$ vs. the Intact group; ${ }^{* *}: p<0.01$ and ${ }^{*}: p<0.05$ vs. the AD Con. 


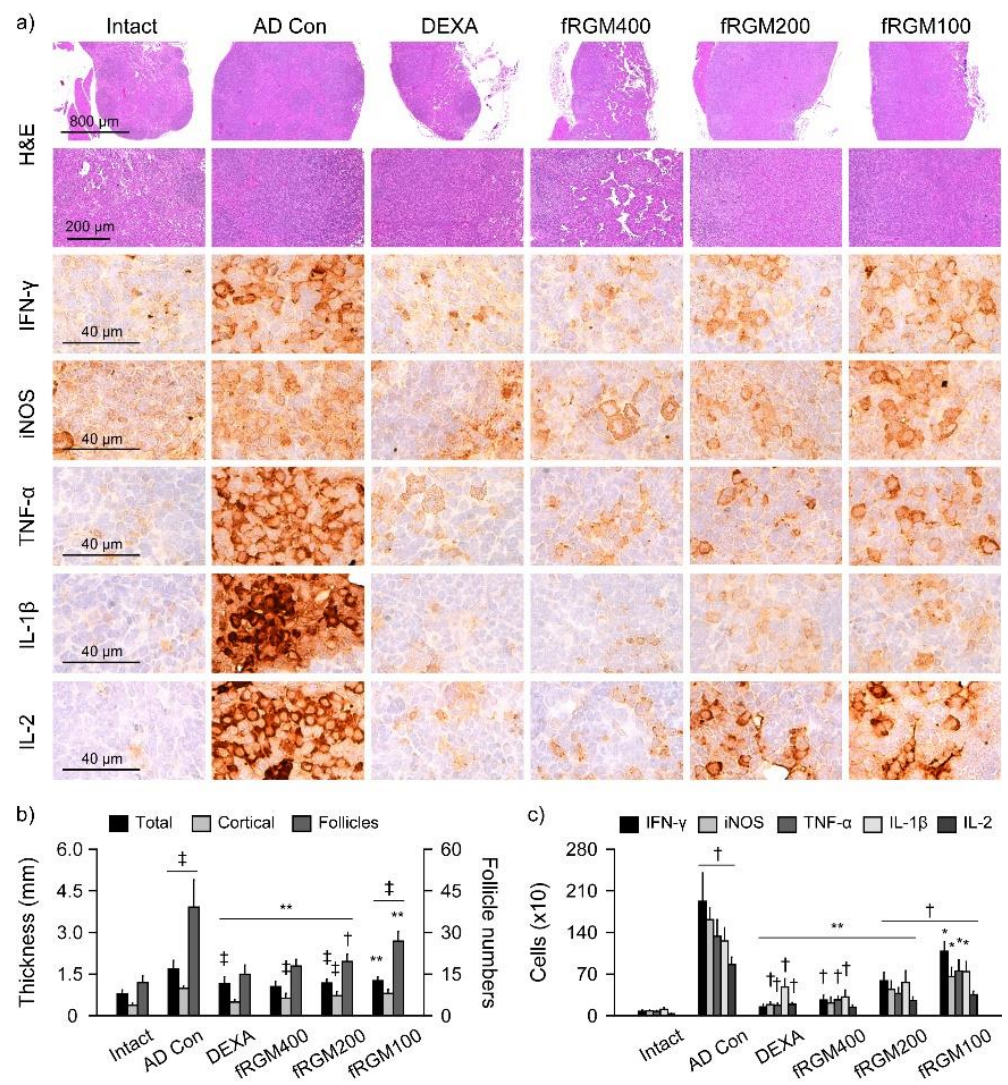

Figure 7. Histopathological changes in the lymph node of AD-like model. (A) Representative images in H\&E stain and immunostains for IFN- $\gamma$, iNOS-2, TNF- $\alpha$, IL-1 $\beta$ and IL-2 in the lymph node. In H\&E stains, the parenchyma in the upper is high-magnified in the lower. (B) Total and cortical thickness in the central region $(\mathrm{mm})$ and number of the cortical follicles (follicles $\left./ \mathrm{mm}^{2}\right)$. (C) Immunoreactive cells in the parenchyma $\left(\times 10\right.$ cells $\left./ \mathrm{mm}^{2}\right)$. Values are represented as the means \pm SDs. $\ddagger: p<0.01$ and $t: p<0.05$ vs. the Intact group; ${ }^{* *}: p<0.01$ and $*: p<0.05$ vs. the AD Con.

\section{Discussion}

Patients with AD generally suffer from chronic pruritus, and scratching worsens skin inflammation by damaging the skin barrier and inducing the production of inflammatory mediators $[28,29]$. In this context, inhibition of clinical symptoms is one of the most important therapeutic strategies for AD. The current AD-like model exhibited AD-like skin lesions and severe scratching; however, fRGM inhibited the clinical symptoms, along with reducing the IgE levels. Elevated IgE and IgE-mediated immune responses involve the characteristic symptoms of AD through allergic inflammation, which are the main hallmarks of AD pathogenesis [29]. In particular, mast cells are major effector cells related to IgE-mediated hypersensitivity reactions, and their activation promotes the production of pro-inflammatory cytokines, together with Th1-derived mediators (i.e., TNF- $\alpha$, IFN- $\gamma$, IL$1 \beta$, IL-6, IL-10) [2,30]. Here, histopathological analyses revealed that fRGM improved ADlike skin lesions by reducing epidermal thickening, collagen deposition and dermal infiltration of inflammatory cells and mast cells. The immunohistochemical analyses suggested the antioxidant, anti-inflammatory and anti-apoptotic effects of the fRGM. These suggest that fRGM still contains the bioactive nutrition involved in anti-AD effects by inhibiting the production of IgE and the subsequent inflammatory progression, similarly with red ginseng even though most of the fRGM is discarded as a byproduct of the red ginseng extraction.

The atopic immune activation is characterized by a Th2-dominated reaction: Th2 cells are responsible for IgE synthesis, and predominantly release IL-4, IL-5, IL-10 and IL-13 [3,31]. Among these cytokines, IL-4 and IL-13 activate mast cells and basophils by promoting IgE isotype switching in B cells and Th2 dominance, and IL-5 and IL-10 involve 
eosinophil activation and the infiltration into the skin lesions, which in turn elicit an allergic immune response [3,32]. Subsequently, chronic AD demonstrates Th1-derived mediators and tissue remodeling with collagen deposition and dermal thickening, leading to further production of IgE and inflammatory mediators by recruiting activated immune cells [30,33]. Here, the AD-like skin lesions up-regulated the mRNA expressions of TNF$\alpha$ and Th2 cytokines including IL-4, IL-5 and IL-13, with increased immunotains for TNF$\alpha$, IFN- $\gamma$, IL-1 $\beta$, IL-2 and iNOS. However, fRGM inhibited the expression of inflammatory mediators, and resulted in mild fibrosis by reducing collagen deposition and expression of MMP-9 as a remodeling marker in patients with AD [34]. The allergic inflammatory response is associated mainly with the activation of intracellular signaling pathways, such as mitogen-activated protein kinases (MAPKs) and nuclear factor kappa B (NF- $\mathrm{B}$ ) [35]. Red ginseng has been reported to exert anti-inflammatory effects via inhibition of the MAPK and NF- $\kappa B$ pathways in AD-like models [36]. RGM, particularly triterpene glycoside compounds, has also shown anti-inflammatory effects by targeting the NF- $\kappa \mathrm{B}$ pathway [21,37]. Further studies are needed to clarify the exact mechanisms by which compounds in the fRGM exert beneficial effects on progression of AD.

The pathophysiology of AD is multi-factorial with immune alteration, skin barrier dysfunction and environmental factors [38]. Skin barrier dysfunction increases the penetration of allergens and pathogens, leading to immune alteration and further disruption of the barrier function [38,39]. The secondary lymphoid tissues including the spleen and lymph nodes are the main regions exposed to various allergens in the chronic stage of AD, which further differentiates naïve $\mathrm{T}$ cells into effector $\mathrm{T}$ cells, and stimulates $\mathrm{B}$ cells to produce IgE [18,40]. Our DNCB-induced AD-like model showed the hypertrophic changes of the spleen and lymph node with the increased inflammatory cytokines in previous and current studies [26]. The changes were involved in hyperplasia of the white pulp cells of the spleen and follicular cells of the lymph node, probably by increasing leukocytes and differentiating into effector lymphocytes after the induction of DNCB [40]. However, fRGM inhibited the systemic immune activation in both lymphoid tissues by inhibiting the production of Th1-derived mediators and the development of the germinal center related to IgE production, suggesting therapeutic potential in chronic AD.

Chronic inflammation of AD elevates free radicals and reactive oxygen and nitrogen species (RONS), similar to other chronic inflammatory diseases, and RONS-generated regional inflammation can further activate allergic responses [41,42]. Here, fRGM improved antioxidant capacities in AD-like skin lesions by increasing antioxidants (GSH) and by reducing lipid peroxidation (MDA and 4-HNE) and superoxide anion, and RONS-induced protein nitration (NT). Natural products generally possess polyphenolic compounds and flavonoids that act as direct free radical scavengers against superoxide anions and inhibitors of lipid peroxidation [18]. Indeed, fRGM contained the polyphenolic compounds and flavonoids despite the small contents, and the antioxidant activities could be enhanced via fermentation with effective microorganisms [22,23,43]. Although the DEXA group had little effect on the skin levels of GSH, MDA and superoxide anions, the potent anti-inflammatory effects may be involved in reducing the RONS-induced products (NT and 4-HNE). Furthermore, the immunostaining for apoptotic markers (Cas-3 and PARP) was reduced in the DEXA and fRGM groups in the AD-like skin lesion, suggesting antiapoptosis related to the anti-inflammatory effects rather than antioxidant effects. However, given that RONS damages DNA and cellular biomolecules, and the most significant target is the MAPK pathway [44], the antioxidant properties of fRGM may contribute to additional anti-allergic effects by inhibiting inflammatory responses and apoptotic changes.

RGM has been considered only a byproduct of ginseng extraction processing; however, recent studies have focused on using RGM as a functional food in human [43] and animal $[45,46]$. Here, topical treatment of fRGM, particularly at higher doses, alleviated the AD-like lesions by inhibiting IgE production, inflammatory progress and apoptotic changes with enhanced antioxidant activities, similar with anti-AD effects of red ginseng. 
These results provide useful information for developing good sources for public health and further increase the market value of RGM in the feed industry.

Supplementary Materials: The following supporting information can be downloaded at: www.mdpi.com/xxx/s1, Figure S1: Organ weights of the spleen and lymph node (LN); Table S1: Primers used for RT-qPCR analysis.

Author Contributions: Conceptualization, Yeun Soo Jung, Jae Young Choi and Chang-Hyun Song; Data curation, Young-Sam Kwon, VinayKumar Dachuri and Sae-Kwang Ku; Formal analysis, GyuRyeul Park, Sae-Kwang Ku and Chang-Hyun Song; Funding acquisition, Sae-Kwang Ku and Chang-Hyun Song; Investigation, Yeun Soo Jung, Jae Young Choi, Sae-Kwang Ku and Chang-Hyun Song; Methodology, Young-Sam Kwon, Young Woo Kim and Sae-Kwang Ku; Project administration, Chang-Hyun Song; Resources, Gyu-Ryeul Park and Chang-Hyun Song; Software, VinayKumar Dachuri; Supervision, Young Woo Kim, Sae-Kwang Ku and Chang-Hyun Song; Validation, Yeun Soo Jung, Jae Young Choi, Sae-Kwang Ku and Chang-Hyun Song; Visualization, Yeun Soo Jung, Jae Young Choi, Young-Sam Kwon, Sae-Kwang Ku and Chang-Hyun Song; Writing - original draft, Yeun Soo Jung and Jae Young Choi; Writing - review \& editing, Young Woo Kim and ChangHyun Song. All authors have read and agreed to the published version of the manuscript.

All authors will be informed about each step of manuscript processing including submission, revision, revision reminder, etc. via emails from our system or assigned Assistant Editor.

Funding: This work was partly supported by the Technology Development Program of MSS (C0237051), the National Research Foundation of Korea (NRF) grant funded by the Korea government (MSIT) (2018R1A5A2025272), and the Basic Science Research Program through the NRF funded by the Ministry of Education (2020R1I1A3A04037939).

Institutional Review Board Statement: The animal study protocol was conducted according to the national regulations of the usage and welfare of laboratory animals based on the NIH guideline, and approved by the Institutional Animal Care and Use Committee of Daegu Haany University (Approval No.: DHU2015-020).

Data Availability Statement: Not applicable here.

Conflicts of Interest: The authors declare no conflict of interest.

\section{References}

1. Lee, J.H.; Son, S.W.; Cho, S.H. A Comprehensive Review of the Treatment of Atopic Eczema. Allergy Asthma Immunol Res 2016, 8, 181-190, doi:10.4168/aair.2016.8.3.181.

2. Lorz, L.R.; Kim, M.Y.; Cho, J.Y. Medicinal potential of Panax ginseng and its ginsenosides in atopic dermatitis treatment. $J$ Ginseng Res 2020, 44, 8-13, doi:10.1016/j.jgr.2018.12.012.

3. Brandt, E.B.; Sivaprasad, U. Th2 Cytokines and Atopic Dermatitis. J Clin Cell Immunol 2011, 2, doi:10.4172/2155-9899.1000110.

4. Leung, D.Y. New insights into atopic dermatitis: role of skin barrier and immune dysregulation. Allergology International 2013, 62, 151-161.

5. Nutten, S. Atopic dermatitis: global epidemiology and risk factors. Ann Nutr Metab 2015, 66 Suppl 1, 8-16, doi:10.1159/000370220.

6. Nygaard, U.; Vestergaard, C.; Deleuran, M. Emerging Treatment Options in Atopic Dermatitis: Systemic Therapies. Dermatology 2017, 233, 344-357, doi:10.1159/000484406.

7. Hussain, Z.; Thu, H.E.; Shuid, A.N.; Kesharwani, P.; Khan, S.; Hussain, F. Phytotherapeutic potential of natural herbal medicines for the treatment of mild-to-severe atopic dermatitis: A review of human clinical studies. Biomed Pharmacother 2017, 93, 596-608, doi:10.1016/j.biopha.2017.06.087.

8. Choi, J.; Kim, T.H.; Choi, T.Y.; Lee, M.S. Ginseng for health care: a systematic review of randomized controlled trials in Korean literature. PLoS One 2013, 8, e59978, doi:10.1371/journal.pone.0059978.

9. Han, M.J.; Kim, D.H. Effects of Red and Fermented Ginseng and Ginsenosides on Allergic Disorders. Biomolecules 2020, 10, doi:10.3390/biom10040634.

10. Kim, H.S.; Kim, D.H.; Kim, B.K.; Yoon, S.K.; Kim, M.H.; Lee, J.Y.; Kim, H.O.; Park, Y.M. Effects of topically applied Korean red ginseng and its genuine constituents on atopic dermatitis-like skin lesions in NC/Nga mice. Int Immunopharmacol 2011, 11, 280285, doi:10.1016/j.intimp.2010.11.022.

11. Sohn, E.H.; Jang, S.A.; Lee, C.H.; Jang, K.H.; Kang, S.C.; Park, H.J.; Pyo, S. Effects of korean red ginseng extract for the treatment of atopic dermatitis-like skin lesions in mice. J Ginseng Res 2011, 35, 479-486, doi:10.5142/jgr.2011.35.4.479.

12. Samukawa, K.; Izumi, Y.; Shiota, M.; Nakao, T.; Osada-Oka, M.; Miura, K.; Iwao, H. Red ginseng inhibits scratching behavior associated with atopic dermatitis in experimental animal models. J Pharmacol Sci 2012, 118, 391-400, doi:10.1254/jphs.11182fp.

13. Osada-Oka, M.; Hirai, S.; Izumi, Y.; Misumi, K.; Samukawa, K.; Tomita, S.; Miura, K.; Minamiyama, Y.; Iwao, H. Red ginseng extracts attenuate skin inflammation in atopic dermatitis through p70 ribosomal protein S6 kinase activation. J Pharmacol Sci 2018, 136, 9-15, doi:10.1016/j.jphs.2017.11.002. 
14. Bae, E.-A.; Han, M.J.; Shin, Y.-W.; Kim, D.-H. Antiallergic and antipsoriatic effects of Korean red ginseng. Journal of Ginseng Research 2005, 29, 80-85.

15. Kim, H.; Park, C.W.; Cho, S.H. The Beneficial Effect of Korean Red Ginseng Extract on Atopic Dermatitis Patients: An 8 Weeks Open, Noncomparative Clinical Study. Ann Dermatol 2018, 30, 304-308, doi:10.5021/ad.2018.30.3.304.

16. Lee, K.G.; Son, S.W. Efficacy of korean red ginseng in the treatment of atopic dermatitis. J Ginseng Res 2011, 35, 149-154, doi:10.5142/jgr.2011.35.2.149.

17. Park, E.K.; Choo, M.K.; Han, M.J.; Kim, D.H. Ginsenoside Rh1 possesses antiallergic and anti-inflammatory activities. Int Arch Allergy Immunol 2004, 133, 113-120, doi:10.1159/000076383.

18. Wu, S.; Pang, Y.; He, Y.; Zhang, X.; Peng, L.; Guo, J.; Zeng, J. A comprehensive review of natural products against atopic dermatitis: Flavonoids, alkaloids, terpenes, glycosides and other compounds. Biomedicine E Pharmacotherapy 2021, 140, 111741, doi:https://doi.org/10.1016/j.biopha.2021.111741.

19. Lee, S.H.; Heo, Y.; Kim, Y.C. Effect of German chamomile oil application on alleviating atopic dermatitis-like immune alterations in mice. Journal of veterinary science 2010, 11, 35-41.

20. Lim, T.S.; Na, K.; Choi, E.M.; Chung, J.Y.; Hwang, J.K. Immunomodulating activities of polysaccharides isolated from Panax ginseng. Journal of medicinal food 2004, 7, 1-6, doi:10.1089/109662004322984626.

21. Chung, I.-M.; Kim, Y.-O.; Ali, M.; Kim, S.-H.; Park, I.; Kim, E.-H.; Yang, Y.-S.; Park, H.-R.; Son, E.-S.; Ahmad, A. Triterpene glycosides from red ginseng marc and their anti-inflammatory activities. Bioorganic $\mathcal{E}$ Medicinal Chemistry Letters 2014, 24, 42034208, doi:https://doi.org/10.1016/j.bmcl.2014.07.042.

22. Eom, S.J.; Hwang, J.E.; Jung, J.; Jee, H.S.; Kim, K.T.; Paik, H.D. Short communication: Antioxidative and antibacterial activities on Staphylococcus aureus and Escherichia coli O157:H4 in milk with added ginseng marc extract fermented by Lactobacillus plantarum KCCM 11613P. J Dairy Sci 2017, 100, 7788-7792, doi:10.3168/jds.2017-12725.

23. Jung, H.-W.; Kim, J.-E.; Seo, J.-H.; Lee, S.-P. Physicochemical and antioxidant properties of red ginseng marc fermented by Bacillus subtilis HA with mugwort powder addition. Journal of the Korean Society of Food Science and Nutrition 2010, 39, 1391-1398.

24. Kim, H.-J.; Chae, I.-G.; Lee, S.-G.; Jeong, H.-J.; Lee, E.-J.; Lee, I.-S. Effects of fermented red ginseng extracts on hyperglycemia in streptozotocin-induced diabetic rats. Journal of Ginseng Research 2010, 34, 104-112.

25. Song, P.H.; Park, G.-R.; Kim, Y.-H.; Jung, D.H.; Ku, S.-K.; Song, C.-H. Hair-Growth-Promoting Effects of Fermented Red Ginseng Marc and Traditional Polyherb Formula in C57BL/6 Mice. Applied Sciences 2021, 11, 1195.

26. Kim, C.G.; Lee, J.E.; Jeong, D.G.; Lee, Y.H.; Park, S.I.; Lee, D.G.; Han, C.H.; Kang, S.J.; Song, C.H.; Choi, S.H.; et al. Bathing effects of east saline groundwater concentrates on allergic (atopic) dermatitis-like skin lesions induced by 2,4-dinitrochlorobenzene in hairless mice. Exp Ther Med 2017, 13, 3448-3466, doi:10.3892/etm.2017.4397.

27. Park, G.; Oh, M.S. Inhibitory effects of Juglans mandshurica leaf on allergic dermatitis-like skin lesions-induced by 2,4dinitrochlorobenzene in mice. Experimental and toxicologic pathology : official journal of the Gesellschaft fur Toxikologische Pathologie 2014, 66, 97-101, doi:10.1016/j.etp.2013.10.001.

28. Mollanazar, N.K.; Smith, P.K.; Yosipovitch, G. Mediators of Chronic Pruritus in Atopic Dermatitis: Getting the Itch Out? Clin Rev Allergy Immunol 2016, 51, 263-292, doi:10.1007/s12016-015-8488-5.

29. Leung, D.Y.; Bieber, T. Atopic dermatitis. Lancet 2003, 361, 151-160, doi:10.1016/S0140-6736(03)12193-9.

30. Kawakami, T.; Ando, T.; Kimura, M.; Wilson, B.S.; Kawakami, Y. Mast cells in atopic dermatitis. Curr Opin Immunol 2009, 21, 666-678, doi:10.1016/j.coi.2009.09.006.

31. Otsuka, A.; Nomura, T.; Rerknimitr, P.; Seidel, J.A.; Honda, T.; Kabashima, K. The interplay between genetic and environmental factors in the pathogenesis of atopic dermatitis. Immunol Rev 2017, 278, 246-262, doi:10.1111/imr.12545.

32. Laouini, D.; Alenius, H.; Bryce, P.; Oettgen, H.; Tsitsikov, E.; Geha, R.S. IL-10 is critical for Th2 responses in a murine model of allergic dermatitis. The Journal of clinical investigation 2003, 112, 1058-1066, doi:10.1172/JCI18246.

33. Galli, S.J.; Tsai, M. Mast cells in allergy and infection: versatile effector and regulatory cells in innate and adaptive immunity. Eur J Immunol 2010, 40, 1843-1851, doi:10.1002/eji.201040559.

34. Devillers, A.C.; van Toorenenbergen, A.W.; Klein Heerenbrink, G.J.; Muldert, P.G.; Oranje, A.P. Elevated levels of plasma matrix metalloproteinase-9 in patients with atopic dermatitis: a pilot study. Clinical and experimental dermatology 2007, 32, 311-313, doi:10.1111/j.1365-2230.2007.02378.x.

35. Arthur, J.S.; Ley, S.C. Mitogen-activated protein kinases in innate immunity. Nat Rev Immunol 2013, 13, 679-692, doi:10.1038/nri3495.

36. Kee, J.Y.; Jeon, Y.D.; Kim, D.S.; Han, Y.H.; Park, J.; Youn, D.H.; Kim, S.J.; Ahn, K.S.; Um, J.Y.; Hong, S.H. Korean Red Ginseng improves atopic dermatitis-like skin lesions by suppressing expression of proinflammatory cytokines and chemokines in vivo and in vitro. J Ginseng Res 2017, 41, 134-143, doi:10.1016/j.jgr.2016.02.003.

37. Bak, M.-J.; Hong, S.-G.; Lee, J.-W.; Jeong, W.-S. Red ginseng marc oil inhibits iNOS and COX-2 via NFkB and p38 pathways in LPS-stimulated RAW 264.7 macrophages. Molecules 2012, 17, 13769-13786, doi:10.3390/molecules171213769.

38. Boguniewicz, M.; Leung, D.Y. Atopic dermatitis: a disease of altered skin barrier and immune dysregulation. Immunol Rev 2011, 242, 233-246, doi:10.1111/j.1600-065X.2011.01027.x.

39. Han, H.; Roan, F.; Ziegler, S.F. The atopic march: current insights into skin barrier dysfunction and epithelial cell-derived cytokines. Immunological reviews 2017, 278, 116-130.

40. Wu, L.C.; Scheerens, H. Targeting IgE production in mice and humans. Curr Opin Immunol 2014, 31, 8-15, doi:10.1016/j.coi.2014.08.001.

41. Bertino, L.; Guarneri, F.; Cannavo, S.P.; Casciaro, M.; Pioggia, G.; Gangemi, S. Oxidative Stress and Atopic Dermatitis. Antioxidants (Basel) 2020, 9, doi:10.3390/antiox9030196. 
42. Briganti, S.; Picardo, M. Antioxidant activity, lipid peroxidation and skin diseases. What's new. Journal of the European Academy of Dermatology and Venereology : JEADV 2003, 17, 663-669.

43. Chung, S.I.; Rico, C.W.; Kang, M.Y. Comparative study on the hypoglycemic and antioxidative effects of fermented paste (doenjang) prepared from soybean and brown rice mixed with rice bran or red ginseng marc in mice fed with high fat diet. Nutrients 2014, 6, 4610-4624, doi:10.3390/nu6104610.

44. Kruk, J.; Duchnik, E. Oxidative stress and skin diseases: possible role of physical activity. Asian Pac J Cancer Prev 2014, 15, 561568, doi:10.7314/apjcp.2014.15.2.561.

45. Ao, X.; Meng, Q.; Kim, I. Effects of fermented red ginseng supplementation on growth performance, apparent nutrient digestibility, blood hematology and meat quality in finishing pigs. Asian-Australasian Journal of Animal Sciences 2011, 24, 525531.

46. Kim, Y.J.; Lee, G.D.; Choi, I.H. Effects of dietary supplementation of red ginseng marc and $\alpha$-tocopherol on the growth performance and meat quality of broiler chicken. Journal of the Science of Food and Agriculture 2014, 94, $1816-1821$. 